
\title{
28 Research Suare \\ Pelargonidin as an Attenuator of Neuronal Mitochondrial Dysfunction: An in Vivo Study
}

Engy Rashed ( $\nabla$ engyrefaat@yahoo.com )

Egyptian Atomic Energy Authority https://orcid.org/0000-0002-6593-378X

Tarek El-Hamoly

NCRRT: National Centre for Radiation Research and Technology

Marwa El-Sheikh

NCRRT: National Centre for Radiation Research and Technology

Mona A. El-Ghazaly

NCRRT: National Centre for Radiation Research and Technology

\section{Research Article}

Keywords: Neuronal mitochondria, PARP inhibition, pelargonidin, neurodegenerative disorders, apoptosis.

Posted Date: April 21st, 2021

DOI: https://doi.org/10.21203/rs.3.rs-407161/v1

License: (9) This work is licensed under a Creative Commons Attribution 4.0 International License. Read Full License 


\section{Abstract}

Targeting the neuronal mitochondria as a possible intervention to guard against neurodegenerative disorders' progression has been investigated in the current work via the administration of Pelargonidin $(\mathrm{PEL})$ to rats intoxicated by the mitochondrial toxin Reserpine. The main criteria for choosing pelargonidin (PEL) were its reported poly (ADP-ribose) polymerase (PARP)-inhibitor, antioxidant, antiapoptotic and anti-inflammatory activities. Male rats were randomized into four experimental groups; normal control, reserpinized to induce mitochondrial failure, standard PARP-1-inhibitor 1,5isoquinolinediol (DIQ)-treated reserpinized, and PEL-treated reserpinized . PEL significantly restored brain glutathione (GSH) with a reduction in nitric oxide contents as compared to reserpine-challenged group. Meanwhile, it improved the neuronal mitochondrial function by the elevation of complex I activity associated with a low ADP/ATP ratio. Likely, through its anti-inflammatory effect, PEL reduced the elevation of serum interlukine-1ß level and inhibited serum lactate dehydrogenase (LDH) activity. These findings were aligned with the reduced expressions of cleaved PARP and cleaved caspase-3 proteins, indicating PEL suppressive effect to the intrinsic apoptotic pathway. Those biochemical findings were confirmed through comparable histopathological tissue examination among the experimental groups. In conclusion, $\mathrm{PEL}$ is a promising candidate for the future use in management of mitochondria-associated neuronal complications via controlling the ongoing inflammatory and degeneration cascades.

\section{Introduction}

Mitochondria are cytoplasmic organelles found in the majority of eukaryotic cells. Commonly, they are 0.75 to $3 \mu \mathrm{m}$ in diameter with different structures and lengths. Intracellular mitochondria form a network that supplies the cellular energy demand via the oxidative phosphorylation (OxPHOS) process. Mitochondrial functions include adenosine triphosphate (ATP) production, intracellular calcium regulation, modulation of the cellular redox status, the release of caspase-activating proteins and free radicals scavenging (Reddy 2007). Being porous, the outer mitochondrial membrane allows the flow of low molecular-weight substances from the cytosolic to the inter-membrane compartments and vice versa. However, the inner mitochondrial membrane is a reliable barrier to the ionic flow, it covers the mitochondrial matrix and houses the electron transport chain (ETC) or the mitochondrial respiratory chain including the tricarboxylic acid (TCA) cycle and the beta-oxidation process (Reddy 2009).

It is widely accepted that the cellular stress induced by progressive inflammation and necrosis is crucially regulated by the pivotal transcriptional factor of the inflammatory response; nuclear factor-KB (NF-KB) (Del Zoppo et al.2000). Subsequently, neurons become more susceptible to cellular death signals provoked by the inflammatory mediators cascade and eventually undergo apoptotic cell death (Liu et al 2004).

Reserpine; a potent natural alkaloid obtained from the roots of a variety of Rauwolfia genus members (Doyle et al 1955). This alkaloid act on depleting the biogenic amines; dopamine, noradrenalin and serotonin, it is also reported as a potent oxidant (Metzger et al 2002). Clinically, reserpine is used as an 
antihypertensive agent with limitations being an inducer of organ oxidative injuries (Al-Bloushi et al 2009).

Poly ADP-ribosyl)ation (PARylation) possess a vital role in many cellular processes; e.g. DNA damage/repair, genes transcription and the maintenance of genomic integrity (Curtin and Szabo 2013). The currently reported poly(ADP-ribose) polymerase (PARP) is an enzymes family whose members catalyze $\mathrm{NAD}^{+}$cleavage into ADP-ribose and nicotinamide moieties, and mediate the polymerization of these moieties on target proteins, with a consequent influences on cellular oxidative homeostasis, metabolic and inflammatory cascades (Bai 2015; Jubin et al 2016). PARP-1 acts by binding tothe nuclear DNA at the points of single-strand break (SSB), select DNA repair molecules then dissociates from the SSB points. The potent PARP inhibitors act via trapping PARP-1 at the site of DNA break, and stabilize the formed PARP-DNA complexes, resulting in DNA double-strand breaks that needs more complex pathways to be repaired (Shen et al 2015). Generally, irradiation or oxidative stress-induced DNA damage results in an over-activation of PARP1, cellular $\mathrm{NAD}^{+}$and ATP depletion, leading to cellular dysfunction followed by necrosis. In addition, mitochondrial DNA (mtDNA) is routienly reacting with reactive species such as reactive nitrogen and oxygen species (RNS and ROS) that are injurious to it; yet, it is constantly repaired through various mechanisms involved in nuclear DNA repair, including PARP1 pathway (Brunyanszki et al 2016). However, NAD depletion and PARP-1 over-activation are reported to be involved in the pathogenesis of many central nervous system (CNS) diseases such as neuro-inflammation, traumatic brain injury (TBI), ischemia/stroke and neurodegenerative diseases whose pathogenesis mainly involves mitochondrial dysfunction including; Alzheimer's (AD), Huntington's disease (HD), Parkinson's diseases (PD), Friedreich's ataxia (FRDA) and amyotrophic lateral sclerosis (ALS) (Wang et al 2019). Thus, the use of PARP inhibitors is supposed to reduce $N A D^{+}$depletion-associated mitochondrial dysfunction and energy failure and to allow the mtDNA repair (Szczesny et al 2014, Krainz et al 2018). In this context, Park et al., (2015) suggested a potential anti-aging effect of the classical PARP-1 inhibitor 1,5-isoquinolinediol (DIQ) via mitochondrial function/energy preservation. On the same line, the anthocyanidin Pelargonidin (PEL) [2-(4-hydroxyphenyl) chromenylium-3,5,7- triol] that is naturally abundant in many fruits and vegetables. Among the structurally-comparable compounds, it has been reported to possess a strong antioxidant effects, a scavenging ability to reactive oxygen species (ROS), and an inhibiting property against lipid peroxidation (Miguel 2017). In addition, PEL was investigated as an anti-inflammatory flavonoid via inhibiting the production of nitric oxide (NO), inducible nitric the expression of oxide synthase (iNOS) and the activity of NF-KB (Hamalainen et al 2007). In vivo, PEL has been studied in a variety of experimental models; viz, atherosclerosis, diabetes and cardiovascular dysfunction (Noda et al 2002; Min et al 2018).

Disease-modifying therapies for neurodegenerative disorders are crucial to slow down the progression of such disorders. Yet, several agents are under current investigation acting via increasing mitochondrial biogenesis, inhibiting apoptosis and attenuating the mitochondria-induced oxidative stress. Thus, the development of a targeted and precise intervention might be pivotal to achieve a disease-modifying therapeutic protocol in the future. The current work was, therefore, designed to investigate the potential 
use of PEL in comparison to a standard PARP-1 inhibitor as a possible disease modifying intervention in neurodegenerative diseases whose pathogenesis involves mitochondrial dysfunction.

\section{Materials And Methods \\ 2.1. Materials}

\subsubsection{Chemicals and Kits}

Reserpine, 1,5-isoquinolinediol, Pelargonidin [2-(4-hydroxyphenyl) chromenylium-3,5,7-triol] were obtained from Sigma-Aldrich Chemical Co. (USA). All other reagents, co-factors and solvent used in this study were of the best analytical grade purchased from the different suppliers. The Enzyme Linked Immunosorbent Assay (ELISA) kit for the determination of interleukin-1ß (IL-1ß) concentration was purchased from My BioSourceßCo., USA. The kinetic kit purchased from Salucea (Haansberg, Netherland) was used to determine lactate dehydrogenase (LDH) activity.

\subsubsection{Animals}

Animals used in the present study were male albino rats of Wistar strain; adults weighing $200 \pm 20$ grams; obtaine from the animal breeding unit of the Egyptian National Research Centre. Prior to the experiment, rats were acclimatized for one week with free access to standard chow diet and tap water. All the experiments performed in this study were according to the instructions detailed of the "Guide for the Care and Use of Laboratory Animals of the National Institutes of Health" (NIH Publications No. 8023, revised 1978).

\subsection{Induction of Neuronal Mitochondrial Dysfunction}

Induction of neuronal mitochondrial dysfunction in rats was carried on by an intraperitoneal (i.p.) injection of reserpine; dissolved in 1\% acetic acid, given at a dose of $5 \mathrm{mg} / \mathrm{kg}$ body weight (b.w.) (Kaur and Starr 1997; Di Marzo et al 2000). To confirm the induction of mitochondrial dysfunction, animals have been inspected by a motor test "grid test"; in brief, the rat was hung by its paws to a vertical grid (44 $\mathrm{cm}$ high and $25.5 \mathrm{~cm}$ wide with a space of $10 \mathrm{~mm}$ between every two wires), the time taken by the rat until its first movement has been recorded (Alam and Schmidt 2004). Rats were then sacrificed $24 \mathrm{~h}$ postreserpine injection (El-Ghazaly et al 2015).

\subsection{Experimental design}

Random assortment of rats into four experimental groups ( $n=8)$ was carried out: $G 1$; control group, where rats received the vehicle of reserpine, G2; reserpine-challenged group, rats were i.p. injected a single dose of $5 \mathrm{mg} / \mathrm{kg}$ in $1 \%$ acetic acid $(1 \mathrm{ml} / \mathrm{kg}), \mathrm{G} 3$; animals received a single dose of PEL (3mg/kg) (Roy et al 2008) one hour after reserpine administration, G4; rats received a single dose of 1,5-isoquinolinediol (DIQ) $(10 \mathrm{mg} / \mathrm{kg}$ ) (Sánchez-Fidalgo et al 2007) one hour after reserpine administration. Animals were sacrificed $24 \mathrm{~h}$ following reserpine administration. Two rats were used for the histological brain tissue examination, while the remaining 6 rats were used for the biochemical assessments. Blood samples were 
collected under light anesthesia prior to their sacrifice while the whole brain samples were dissected out and removed for later histopathological examinations and biochemical analysis.

\subsection{Biochemical assays}

2.4.1. Assessment of brain oxidative stress biomarkers: Brain tissue contents of each of the total nitrate/nitrite (NOx) and the reduced glutathione (GSH) was measured following the methods of Miranda et al (2001) and Beutler et al (1963) respectively.

2.4.2. Determination of serum LDH activity and IL-1ß level: The serum level of IL-1ß was determined according to the supplier's insert of an enzyme linked immune-sorbent assay (ELISA) kits. As a marker of cellular integrity; serum LDH activity was assessed by kinetic enzymatic kit according to the kit manufacturer's instructions.

2.4.3. Assessment of mitochondrial functions: Brain mitochondrial fractions were prepared using the fractionation protocol described by Turpeenoja et al (1988). The ADP and ATP contents were acid/base extracted from the mitochondrial fractions according to the method of Glick et al (1993) and quantified by high performance liquid chromatography (HPLC) based on the method of Harmsen et al (1982). The HPLC system was equipped with an ultraviolet (UV) detector (Sykam, Germany) and was set based on the separation conditions using a C18 column (Hypersil, USA) with $0.5 \mathrm{ml} / \mathrm{min}$ flow rate at $25^{\circ} \mathrm{C}$, an injection volume of $20 \mu \mathrm{l}$ and run time of $10 \mathrm{~min}$. The mobile phase components were $0.2 \mathrm{M} \mathrm{KH}_{2} \mathrm{PO}_{4}$ : acetonitrile: methanol in ratios 9.6:0.3:0.1. Peaks were detected at $\lambda=254 \mathrm{~nm}$. Then ATP concentration was calculated from a constructed calibration curve. The activity of the mitochondrial complex-I (Cplx-I) was determined by the method described by Whitfield et al (1981) based on the reaction catalyzed by Cplx-I by following the decrease in absorbance due to NADH oxidation with the ubiquinone-1 (Coenzyme-Q1) reduction into ubiquinol-1 at $340 \mathrm{~nm}$.

2.4.4. Western blotting analysis: Quantitative estimations of Cleaved Caspase-3 and Cleaved PARP were assessed by western blotting analysis. Sample preparation and immune-blotting was performed as described by Mingone et al (2003). In brief; about $5 \mathrm{mg}$ piece of frozen brain tissue were homogenized with $300 \mu \mathrm{l}$ Radioimuno-precipitation assay (RIPA) buffer, consisting of $20 \mathrm{mM}$ Tris-HCL pH7.4 + 150mM $\mathrm{NaCl}+1 \mathrm{mM}$ EDTA $+1 \%$ Triton-X100 $+1 \%$ sodium deoxycholate $+0.1 \%$ SDS. Total tissue protein contents were measued using BCA kit (Pierce, Rockford, USA). A sample of twenty micrograms of denatured protein was run on $8 \%$ and $15 \%$ SDS-PAGE then transferred onto nitrocellulose membrane for the detection of PARP-1/cleaved-PARP and caspase-3; respectively. Non-specific protein binding was blocked by $5 \% \mathrm{w} / \mathrm{v}$ BSA in TBS-T as an incubation media for the membranes. Thereafter, membranes were incubated with polyclonal rabbit anti-PARP-1 antibody (cell signal, MI, USA), polyclonal rabbit anti-cleaved caspase-3 antibody (cell signal, MI, USA) at dilutions of 1:1000 and 1:500, respectively. After washing the excess primary antibody, membranes were incubated with HRP-conjugated anti-rabbit antibodies. All blots were normalized to monoclonal mouse anti- $\beta$-actin (Sigma-Aldrich, St. Louis, USA). Protein bands were detected using ECL Plus kit (Pierce, Rockford, USA), then their densitometry was analyzed by using $\mathrm{NIH}$ image $\mathrm{J}{ }^{\circledR}$ analyzer software. 
2.5. Histopathological tissue examination: Whole brains of the studied groups were separated, fixed in neutral $10 \%$ formalin solution. Tissue samples were then dehydrated in serial concentrations of ethanol, cleared in xylene, embedded in paraffin wax then sliced into 5-micron thickness sections. Fixed sections were stained using hematoxylin and eosin (H\&E) stain for examination using light microscope according to the method of Bancroft et al (1996).

2.6. Data analysis: Statistical analysis of the observed data was performed by one-way analysis of variance (ANOVA) test, followed by Tukey-Kramer post-test for multiple comparisons among different groups' means using the software; GraphPad version 6, La Jolla, CA. The results presented were expressed as means \pm standard error of the means (SEM). Differences were set to be significant at $p<$ 0.05 .

\section{Results}

\subsection{Pelargonidin modifies the unbalanced oxidative status and nitric oxide level triggered in reserpine-challenged rats:}

Excessive ROS and RNS production are check point for cellular damage. Reserpine administration resulted in marked decrease in reduced GSH (a marker of anti-oxidant capacity) with an elevated nitric oxide contents as compared to the control group. Treatment with either PEL or DIQ significantly decreased the elevated levels of nitric oxide contents. On the other hand, DIQ did not exert a remarkable correction of the decreased GSH concentration (Fig. 1a, b).

\subsection{Pelargonidin ameliorates the inflammatory response and cellular integrity damage triggered in reserpine- challenged rats:}

As shown in Fig. 2a, severe inflammatory response was observed in reserpine-challenged rats as indicated by the remarkable up-leveling of the serum proinflammatory IL-1ß concentration.

Administration of either DIQ or PEL significantly attenuated the elevation in serum cytokine concentration in reserpine-challenged rats. Increased plasma membrane permeability following reserpine administration was indicated by the elevation in serum LDH activity; a sign of necrotic tissue formation. Meanwhile, a pronounced LDH release into the circulation was elucidated in reserpine-challenged rats. Each of DIQ and PEL-treated rats showed significant decrease in LDH serum-activity in comparison to the untreated ones (Fig. 2b).

\subsection{Pelargonidin corrects the mitochondrial complex-I activity and ADP/ATP ratio in reserpine-challenged rats:}

In the present study, reserpine administration accompanied by disturbed mitochondrial function as well as excessive energy consumption, indicated by lower complex I activity and higher ADP/ATP ratio. 


\subsection{Pelargonidin inhibits the aggravated apoptotic cascade in reserpine-challenged rats:}

Based on the densitometry analysis of the western blot bands, striatal cleaved caspase-3 and cleavedPARP contents were significantly increased following reserpine administration, amounting to to 360 and $450 \%$ of the control group, respectively. Meanwhile, each of DIQ and PEL inhibited these increments to 47 and $54 \%$, respectively, in comparison to the reserpine-challenged group (Fig. 4).

\subsection{Pelargonidin corrects the brain histopathologic changes in reserpine-challenged rats:}

The histological tissue examinations revealed that substantia nigra area in control rats consisted of perikaryon cells with short shrunken dendrites. Their cell bodies showed branching pattern of the dendrites. Three thin dendrites emerging from the perikaryon were obvious. As for the the distal dendntic parts, they showed axon-like filiform processes [Fig. 5a]. The examination also revealed the presence of medium-sized fusiform, ovoid and pyramidal neurons mainly localized in the pars compacta and pars reticulate areas [Fig. 5b]. Lewy bodies (- =absent or not discernible). reserpine-challenged rats animals group showed dopaminergic neuronal loss from the substantia nigra. Degeneration and necrosis of nigral neurons were seen. Neurodegeneration was accompanied by reactive changes such as astrogliosis and microglial cellular activation [Fig. 5c, d]. In addition, the injured cortical areas showed neuronal loss, gliosis and spongiosis, with ballooned neurons. Interestingly, Lewy bodies appeared as pale, granular bodies with ill-defined rounded areas of granular, pale eosinophilic substance [Fig. 5e]. In case of neuronal death, Lewy bodies were detected as extracellular deposits in the neurophil [Fig. 5f] Lewy bodies (+++ = severe). Animals group treated with QIA showed mild neuronal degeneration of substantia nigra neurons with gliosis in comparison to the reserpine-challenged group. Ballooning of neurons and shorting of dendrites were also observed [Fig. 5g]. Lewy bodies were detected in both inter- and intra-neuronal areas. Scarce losses in neuronal substantia nigra especially in the ventrolateral neurons of the pars Compacta were noticed, in addition to Lewy bodies (++ = moderate). PEL treated-group treated with PEL showed mild neuronal degeneration in substantia nigra [Fig. 5h]. Very few number of cellular Lewy bodies (+ = slight) were seen [Fig. 5i].

\section{Discussion}

Several cellular and molecular mechanisms are involved in the pathogenesis and/or the progression in a number of neurodegenerative disorders (ND), including oxidative/ nitrosative stress, up regulation of inflammatory cytokines, mitochondrial dysfunction, PARylation and different forms of cell demises; apoptosis or necrosis. Thus, targeting the neuronal mitochondria as a possible intervention to guard 
against ND progression has been studied in the present work via the administration of PEL to rats intoxicated by the mitochondrial toxin Reserpine.

In general, mitochondrion is the main source of ROS through the respiratory chain complexes activities. The natural compensatory mechanisms can get rid of the ROS and neutralize their peroxides; detoxifies the superoxide anion into hydrogen peroxide which is then captured by glutathione peroxidase or catalase consuming GSH (Jiang et al 2016) These facts were reflected in the present work; whereby the antioxidant mechanisms were impaired by the production of reserpine-derived oxidants with a consequent depletion of brain GSH contents as compared to control.

In the present work, PEL administration significantly enhanced the antioxidant capacity characterized by marked elevation of reduced GSH accompanied with a remarkable reduction in nitric oxide formation indicated by lower nitrite content. Similar PEL effects were reported by Roghani et al (2010)]; in their study, administration of PEL offered a neuroprotective effect in 6-hydroxydopamine (6-OHDA)-induced neurotoxicity, partly through the inhibition of lipid peroxidation, activating the antioxidant enzyme SOD and lowering nitric oxide/free radicals' production. Moreover, PEL has been reported to protect against the progression of the memory dysfunction in an amyloid-induced Alzheimer's disease in rats via inhibiting the oxidative and nitrosative stress (Sohanaki et al 2016)

Furthermore herein; the PARP-1 inhibitor DIQ inhibited the reserpine-induced rise in the nitric oxide production and brain GSH content depletion. Numerous studies have reported the involvement of PARylation in various pathological conditions; for instance, detection of poly (ADP-ribose) accumulation reflects the PARP activation in stroke (Martire et al 2015), myocardial infarction (Halmosi et al 2001) and neurodegenerative diseases, like PD and AD (Martire et al 2015). Since superoxide and nitric oxide need to be produced in almost equimolar amounts to form peroxynitrite, PARP inhibition has been reported to inhibit the production of both free radicals may result in suppressed peroxynitrite-dependent lipids peroxidation, pro-inflammatory cytokines expression and DNA breaks formation (Virág and Szabó 2002); which might explain the anti-inflammatory and anti-apoptotic actions of DIQ observed in the present study.

On the same line with the present findings, reserpine administration induced neuronal death; either through the activation of apoptotic cascade or via the induction of necroptosis as indicated by the elevation serum LDH activity; where the occurrence of different modes of cell death is characteristic for neurodegenerative disorders (Ghavami et al 2014). It is well-established that NF-kB stimulates the upregulation of iNOS activity, increasing NO- production consequently (Pall 2008). Moreover, the fact that both $\mathrm{NO} \cdot$ and peroxynitrite are significant mediators of cellular injuries in various cases of inflammation suggests that the PARP-related apoptosis plays a main role in diseases in which oxidative stress and/or inflammation are involved. Therefore, PEL-induced inhibition of ROS and pro-inflammatory cytokines over-production might be the mechanism through which PEL exerted its anti-apoptotic and antiinflammatory actions observed herein. Likewise, the anti-inflammatory effects of PEL has been reported in a mouse sepsis model; while PEL suppressed the tumor growth factor-beta (TGF- $\beta$ )-induced blood 
brain barrier disruption, the cell adhesion molecules and pro-inflammatory cytokines expressions as well as the neutrophils and leukocytes migrations (Jeong et al 2017). In the same context, PEL-induced NF-KB suppression might have slowed down the activation signals of the inflammatory cascade as indicated by the decrement of serum IL-1 $\beta$ concentration. In harmony, PEL has been proven to attenuate LPS-induced NF-KB activation in Murine J774 macrophages (Hamalainen et al 2007), to suppress the cytokines production in a sepsis model in mouse (Lee and Bae 2019) and to inhibit the degradation of the signaling protein $\mathrm{IKB}$, thus attenuating the NF-KB-dependent genes expression in a rat model of $A D$ (Sohanaki et al 2016). This could be explained by a compensatory mechanism against the inflammatory background resulting of the injury.

In the present study, mitochondrial damage was induced by reserpine administration and was accompanied by the activation of caspase-3 (cleaved caspase-3) and breaking down DNA-repairing system (cleaved PARP-1), suggesting that it is involved in neuronal demise. PEL administration significantly attenuated the expression of each of cleaved-PARP-1 and cleaved-caspase-3. In the same context with the present outcomes, the study of Škemienè et al (2013) has demonstrated that pretreatment with PEL offered myocardial against ischemia/reperfusion-induced apoptosis and neurons with an evidence of inhibiting the expressions of pro-apoptotic proteins and serum LDH activity. In addition, a relevant study reported the PEL capability to inhibit caspase activation in a hyperglycemic in vitro model challenged with mitochondrial perturbation (Samadder et al 2017). Besides, IL-1 $\beta$ has been reported to offer a significant pro-apoptotic action in serum deprivation conditions, to enhance the mitochondrial cytochrome-c release into the cytosol and to lower the Bcl-2/Bax ratio; verifying that the mitochondria-mediated apoptotic pathway is augmented under inflammatory status (Shen et al 2015). In harmony with the current observations, variable degrees of histological neuronal degenerations were observed in brain tissue of reserpine-challenged rats. Similar lesions were more or less reported in previous studies (Rogers et al 2007). The administration of PEL protected the brain tissue against reserpine-induced neuronal injuries. Similar observations were observed in DIQ treated rats.

As for the cellular energetics, their imbalance/failure has been reported to induce cellular dysfunction or even death. On the same line, oxidative stress radically triggers DNA apparitions and ATP depletion on the expense of DNA repair mechanisms, a process that ends up by apoptosis. In addition, ROS-induced DNA breakdown induces PARP over activation with a consequent cellular NAD+/ATP depletion accompanied by mitochondrial ATP depletion and cell death promotion (Virág and Szabó 2002). These ROS-induced events were indicated in the present work, whereas the administration of reserpine induced a marked mitochondrial ATP depletion and this effect effectively attenuated by the inhibition of PARP. Furthermore, such events were also attenuated upon PEL administration; where PEL conserved the mitochondrial ATP contents and regulated the mitochondrial function as indicated by the elevated complex I activity. Moreover, former studies have reported the capability of PEL to preserve the mitochondrial membranes integrity so as to maintain its vital function of energy production as PEL-treated group showed an increment of brain ATP content, and to exert beneficial role on hyperglycemia-linked mitochondrial dysfunction (Samadder et al 2017, Su et al 2020). One possible explanation is via CD38 inhibition by PEL and other chemically related flavonoids (Ruan et al 2018) that might contribute in a compensatory 
autophagic flux via CD38/cyclic ADP ribose/autophagy pathway. Meanwhile, the injured mitochondriamediated autophagy protected against apoptosis, suggesting the activation of a positive feedback loop under excitatory stresses (Zhang et al 2020) Aforementioned bioenergetic modulatory effect linked to $P E L$, is in line with its previous plethora on different pathological conditions; cancers and AD. In conclusion, our findings indicated the effectiveness of PEL as a mitochondrial function(s) booster at different levels of proposed mechanisms thus could be a promising candidate for the partial management of mitochondria-associated neuronal complications via controlling the ongoing inflammatory and degeneration cascades.

\section{Declarations}

Acknowledgement: The authors acknowledge Prof. Dr. Ahmed Othman; Professor of Histopathology, Faculty of Veterinary Medicine, Cairo University for his efforts in the histopathological tissue examination and interpretation in the present work.

Funding: This research did not receive any specific grant from funding agencies in the public, commercial, or not-for-profit sectors.

Competing interests: Authors declares no conflicts of interests among them.

Availability of data and materials: All the data/materials related to this study are included in the submitted files.

Code availability: No related codes

Authors' contributions: ER and TH designed the experimental protocol. MM and ER conducted the practical work including animals handling procedures and analytic assays. GR contributed new reagents or analytical tools. MG and MM analyzed the data and drafted the manuscript. TH edited and revised the manuscript. All authors read and approved the manuscript.

Ethics approval: All the animal-involving procedures in this work were carried out in accordance to the guidelines of the "Guide for the Care and Use of Laboratory Animals of the National Institutes of Health" (NIH Publications No. 8023, revised 1978).

Consent to participate/ Consent for publication: No human experiments are included.

\section{References}

1. Alam M, Schmidt WJ (2004) L-DOPA reverses the hypokinetic behaviour and rigidity in rotenonetreated rats. Brain Res. 153,439-446. doi: 10.1016/j.bbr.2003.12.021.

2. Al-Bloushi S, Safer AM, Afzal M, Mousa SA (2009) Green tea modulates reserpine toxicity in animal models. J. Toxicol. Sci. 34(1),77-87. 
3. Bai P (2015) Biology of Poly (ADP-Ribose) Polymerases: The Factotums of Cell Maintenance, MolCel. 58, 947-958. doi: 10.1016/j.molcel.2015.01.034

4. Bancroft JD, Stevens A, Turner DR (1996) Theory and practice of histological technique. Edinburgh: 4th ed. New York: Churchill Livingston. 273-292.

5. Beutler E, Duron O, Kelly BM (1963) Improved method for the determination of blood glutathione. J Lab Clin Med. 61: 882-888.

6. Brunyanszki A, Szczesny B, Virag L, Szabo C (2016) Mitochondrial poly(ADP-ribose) polymerase: The Wizard of Oz at work, Free RadicBiol Med. 100, 257-270.doi: 0.1016/j.freeradbiomed.2016.02.024.

7. Curtin NJ, Szabo C (2013) Therapeutic applications of PARP inhibitors: anticancer therapy and beyond. Mol Aspects Med. 34(6), 1217-1256. doi: 1016/j.mam.2013.01.006

8. Del Zoppo G, Ginis I, Hallenbeck JM, ladecola C, Wang X, Feuerstein GZ(2000) Inflammation and stroke: putative role for cytokines, adhesion molecules and iNOS in brain response to ischemia. Brain Pathol. 10(1) 95-112. doi: 10.1111/j.1750-3639.2000.tb00247

9. Di Marzo V, Hill MP, Bisogno T, Crossman AR, Brotchie JM (2000) Enhanced levels of endogenous cannabinoids in the globuspallidus are associated with a reduction in movement in an animal model of Parkinson's disease. FASEB J. 14(10), 1432-1438. doi: 10.1096/fj.14.10.1432.

10. Doyle AE, McQueen EG, Smirk FH (1955) Treatment of hypertension with reserpine, with reserpine in combination with pentapyrrolidinium, and with reserpine in combination with veratrum alkaloids. Circulation, 11, 170-181. doi: 10.1161/01.cir.11.2.170.

11. El-Ghazaly MA, Sadik NA, Rashed ER, Abd-El-Fattah AA (2015) Neuroprotective effect of EGb761® and low-dose whole-body y-irradiation in a rat model of Parkinson's disease. Toxicol Ind Health. 31(12),1128-1143. doi: 10.1177/0748233713487251.

12. Ghavami S, Shojaei Y, Yeganeh B, Ande SR, Jangamreddy JR, Mehrpour M, Hashemi M (2014) Autophagy and apoptosis dysfunction in neurodegenerative disorders. Prog. Neurobiol.112, 24-49. doi: 10.1016/j.pneurobio.2013.10.004.

13. Glick BS, Wachter C, Reid GA, Schatz G (1993) Import of cytochrome b2 to the mitochondrial intermembrane space: the tightly folded heme-binding domain makes import dependent upon matrix ATP. Prot. Sci. 2 ,1901-1917. doi: 1002/pro.5560021112

14. Halmosi R, Berente Z, Osz E, K. Toth, Literati-Nagy P, Sumegi B (2001) Effect of poly(ADP-ribose) polymerase inhibitors on the ischemia-reperfusion-induced oxidative cell damage and mitochondrial metabolism in Langendorff heart perfusion system. Pharmacol. 59 :1497-1505. doi: 10.1124/mol.59.6.1497.

15. Hamalainen M, Nieminen R, Vuorela P, Heinonen M, Moilanen E (2007) Anti-inflammatory effects of flavonoids: genistein, kaempferol, quercetin, and daidzein inhibit STAT-1 and NFkappaB activations, whereas flavone, isorhamnetin, naringenin, and pelargonidin inhibit only NF-kappa B activation along with their inhibitory effect on iNOS expression and NO production in activated macrophages. Mediators Inflamm., 45673. doi: 10.1155/2007/45673. 
16. Harmsen E, De Tombe P Ph, De Jong JW (1982) Simultaneous determination of myocardial adenine nucleotides and creatine phosphate by high-performance liquid chromatography. J Chromatogr; 230 , 131-136. doi: 10.1016/s0378-4347(00)81439-5.

17. Jeong S, Ku SK, Bae JS (2017) Anti-inflammatory effects of pelargonidin on TGFBIp-induced responses.Can. J. Physiol. Pharmacol. 95(4), 372-381. doi: 10.1139/cjpp-2016-0322

18. Jiang T, Sun Q, Chen S (2016) Oxidative stress: a major pathogenesis and potential therapeutic target of antioxidative agents in Parkinson's disease and Alzheimer's disease. Prog. Neurobiol. 147, 1-19. doi: 10.1016/j.pneurobio.2016.07.005.

19. Jubin T, Kadam A, Jariwala M, Bhatt S, Sutariya S, Gani AR, Gautam S, Begum R (2016) The PARP family: insights into functional aspects of poly (ADP-ribose) polymerase-1 in cell growth and survival, Cell Prolif $49,421-437$. doi: 1111/cpr.12268

20. Kaur S, Starr MS (1997) Differential effects of intrastriatal and intranigral injections of glutamate antagonists on motor behaviour in the reserpine-treated rat. Neuroscience.76(2), 345-354. org/10.1016/S0306-4522(96)00407-1

21. Krainz T, Lamade AM, Du L, Maskrey TS, Calderon MJ, Watkins SC, Clark RS (2018) Synthesis and evaluation of a mitochondria-targeting Poly (ADP-ribose) Polymerase-1 inhibitor. ACS Chem. Biol. 13(10), 2868-2879. doi: 1021/acschembio.8b00423

22. Lee IC, Bae JS (2019) Pelargonidin protects against renal injury in a mouse model of sepsis. J. Med. Food. 22(1), 57-61.org/10.1089/jmf.2018.4230.

23. Liu WG, Chen Y, Li B, Lu GQ, Chen SD (2004) Neuroprotection by pergolide against levodopa-induced cytotoxicity of neural stem cells.Neurochem Res 29(12):2207-2214. doi: 10.1016/j.parkreldis.2006.07.015.

24. Martire S, Mosca L, d'Erme M (2015) PARP-1 involvement in neurodegeneration: A focus on Alzheimer's and Parkinson's diseases, Mech Ageing Dev. 146-148, 53-64. doi: 10.1016/j.mad.2015.04.001

25. Metzger RR, Brown JM, Sandoval V, Rau KS, Elwan MA, Miller GW, Hanson GR, Fleckenstein AF (2002) Inhibitory effect of reserpine on dopamine transporter function. Eur. J. Pharmacol. 456,39-43.

26. Miguel MG (2011) Anthocyanins: Antioxidant and/or anti-inflammatory activities. J. Appl. Pharm. Sci. 1(6), 7-15. doi: 10.1016/s0014-2999(02)02647-x.

27. Min YS, Yoon HJ, Je HD, Lee JH, Yoo SS, Shim HS, Sohn UD (2018) Endothelium independent effect of pelargonidin on vasoconstriction in rat aorta.BiomolTher. 26(4) 374. doi: 10.4062/biomolther.2017.197

28. Mingone CJ, Gupte SA, Quan S, Abraham NG, Wolin MS (2003) Influence of heme and heme oxygenase- 1 transfection of pulmonary microvascular endothelium on oxidant generation and cGMP.Exp. Biol. Med. 228(5), 535-539. org/10.1177/15353702-0322805-22

29. Miranda KM, Espey MG, Wink DA (2001) A rapid, simple spectrophotometric method for simultaneous detection of nitrate and nitrite.Nitricoxide-Biol. Ch.5, 62-71. doi: 10.1006/niox.2000.0319. 
30. Noda Y, Kaneyuki T, Mori A, Packer L (2002) Antioxidant activities of pomegranate fruit extract and its anthocyanidins: delphinidin, cyanidin, and pelargonidin. J. Agric. Food Chem. 50(1), 166-171. doi: 10.1021/jf0108765

31. Pall ML (2008) Post-radiation syndrome as a NO/ONOO- cycle, chronic fatigue syndrome-like disease. Med Hypotheses. 71:537-541. doi: 10.1016/j.mehy.2008.05.023

32. Park MS, Choi JS, Lee W, Yang Y, Kim J, Lee G, Choi JW (2015) Pharmacogenomic analysis indicates potential of 1,5-isoquinolinediol as a universal anti-aging agent for different tissues. Oncotarget. 6(19) 17251. doi: 10.18632/oncotarget.3949.

33. Reddy PH (2007) Mitochondrial dysfunction in aging and Alzheimer's disease: strategies to protect neurons. Antioxid Redox Signal 9:1647-1658. doi: 10.1089/ars.2007.1754

34. Reddy PH (2009) The role of mitochondria in neurodegenerative diseases: mitochondria as a therapeutic target in Alzheimer's disease. CNS Spectr.14(8 Suppl 7): 8-18.

35. Rogers J, Mastroeni D, Leonard B, Joyce J, Grover A (2007) Neuroinflammation in Alzheimer's disease and Parkinson's disease: are microglia pathogenic in either disorder? Int Rev Neurobiol 82 : 235-246. doi: 10.1016/S0074-7742(07)82012-5

36. Roghani M, Niknam A, Jalali-Nadoushan MR, Kiasalari Z, Khalili M, Baluchnejadmojarad T (2010). Oral pelargonidin exerts dose-dependent neuroprotection in 6-hydroxydopamine rat model of hemiparkinsonism. Brain Bull. 82(5-6), 279-283. doi: 10.1016/j.brainresbull.2010.06.004.

37. Roy M, Sen S, Chakraborti AS (2008) Action of pelargonidin on hyperglycemia and oxidative damage in diabetic rats: implication for glycation-induced hemoglobin modification. Life Sci. $82,1102-1110$. doi: 10.1016/j.Ifs.2008.03.011.

38. Ruan Q, Ruan J, Zhang W, Qian F, Yu Z (2018) Targeting NAD+ degradation: The therapeutic potential of flavonoids for Alzheimer's disease and cognitive frailty. Pharmacol. Res. 128 , 345-358.doi: 10.1016/j.phrs.2017.08.010

39. Samadder A, Tarafdar D, Abraham SK, Ghosh K, Khuda-Bukhsh AR (2017) Nano-pelargonidin protects hyperglycemic-induced L6 cells against mitochondrial dysfunction. Plantamedica, 83(05) ,468-475. doi: 10.1055/s-0043-100017.

40. Sánchez-Fidalgo S, Villegas I, Martín A, Sánchez-Hidalgo M, de la Lastra CA (2007) PARP inhibition reduces acute colonic inflammation in rats. Eur. J. Pharmacol. 563(1-3) ,216-223. doi: 10.1016/j.ejphar.2007.01.070

41. Shen Y, Aoyagi-Scharber M, Wang B (2015) Trapping Poly (ADP-Ribose) Polymerase, J Pharmacol Exp Ther. 353, 446-457.doi: 10.1124/jpet.114.222448

42. Škèmienė K, Jablonskienė G, Liobikas J, Borutaitè V(2013) Protecting the heart against ischemia/reperfusion-induced necrosis and apoptosis: the effect of anthocyanins. Medicina, 49(2) , doi: 10.3390/medicina49020015.

43. Sohanaki H, Baluchnejadmojarad T, Nikbakht F, Roghani M (2016) Pelargonidin improves memory deficit in amyloid $\beta 25-35$ rat model of Alzheimer's disease by inhibition of glial activation, 
cholinesterase, and oxidative stress. Biomed Phamacother. 83, 85-91. doi:

10.1016/j.biopha.2016.06.021

44. Su H, Xie L, Xu Y, Ke H, Bao T, Li Y, Chen W(2020) Pelargonidin-3-O-glucoside derived from wild raspberry exerts antihyperglycemic effect by inducing autophagy and modulating gut microbiota. J. Agric. Food Chem. 18;68(46) :13025-13037. doi: 10.1021/acs.jafc.9b03338.

45. Szczesny B, Brunyanszki A, Olah G, Mitra S, Szabo C (2014) Opposing roles of mitochondrial and nuclear PARP1 in the regulation of mitochondrial and nuclear DNA integrity: implications for the regulation of mitochondrial function. Nucleic Acids Res.42(21), 13161-13173. doi: 10.1093/nar/gku1089.

46. Turpeenoja L, Villa RF, Magri G, Giuffrida Stella A M (1988) Changes of mitochondrial membrane proteins in rat cerebellum during aging. Neurochem. Res., 13 (9):859-865. doi: 10.1007/BF00970754

47. Virág L, Szabó C (2002) The therapeutic potential of poly (ADP-ribose) polymerase inhibitors. Pharmacol Rev. 54 :375-429. doi: 10.1124/pr.54.3.375.

48. Wang Y, Xu E, Musich PR, Lin F (2019) Mitochondrial dysfunction in neurodegenerative diseases and the potential countermeasure. CNS Neurosci Ther. 25: 816-824. doi: 10.1111/cns.13116.

49. Whitfield R, Bostedor D, Goodrum M, Haak EH (1981) Chu Hamster cell mutants unable to grow on galactose and exhibiting an overlapping complementation pattern are defective in the electron transport chain. J. Biol. Chem., 256 (13) : 6651-6656.

50. Zhang X, Li L, Zhang Q, Wei Q, Lin J, Jia J, Zhang P (2020) CD38 Causes Autophagic Flux Inhibition and Cardiac Dysfunction through a Transcriptional Inhibition Pathway Under Hypoxia/Ischemia Conditions. Front. Cell Dev. Biol. 8:191. doi.org/10.3389/fcell.2020.00191.

\section{Figures}


(a)

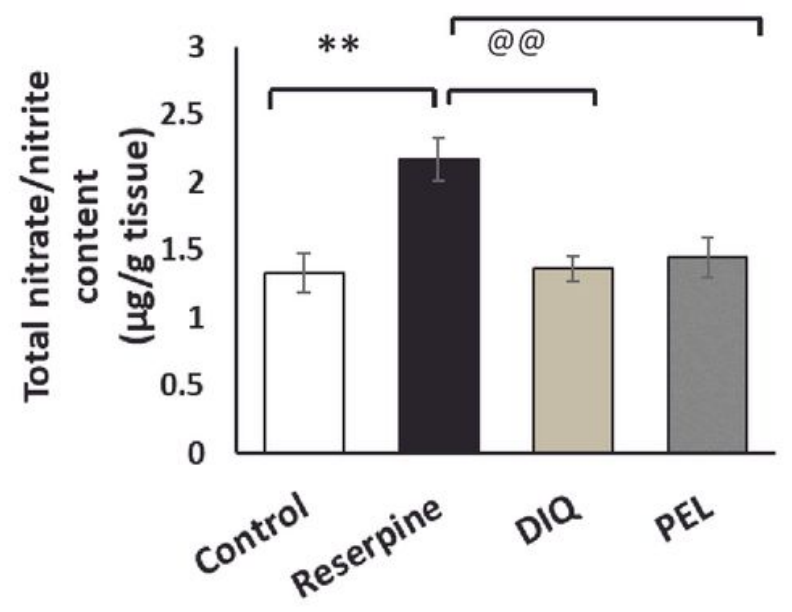

(b)

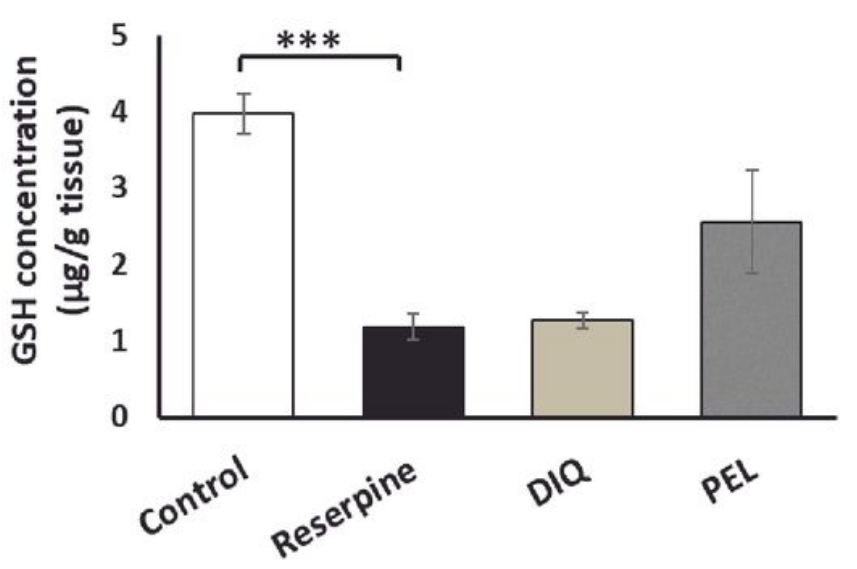

\section{Figure 1}

Effect of each of reserpine (5mg/ $\mathrm{kg}$ ), pelargonidin (PEL) $(3 \mathrm{mg} / \mathrm{kg}$ ) and I,5-isoquinolinediol (DIQ) (10 $\mathrm{mg} / \mathrm{kg}$ ) on brain contents of (a) Nitrite and (b) Reduced glutathione (GSH) in male rats. Results are represented as means of 6 observations per group \pm SEM. $\left.{ }^{(* *}\right)$ and $\left({ }^{\star * *}\right)$ denote significant difference from the control group at $p<0.01$ and p $<0.001$; respectively. (\#) and (@@) denote significant difference from the reserpine-challenged group at $p<0.05$ and $p<0.01$; respectively 
(a)

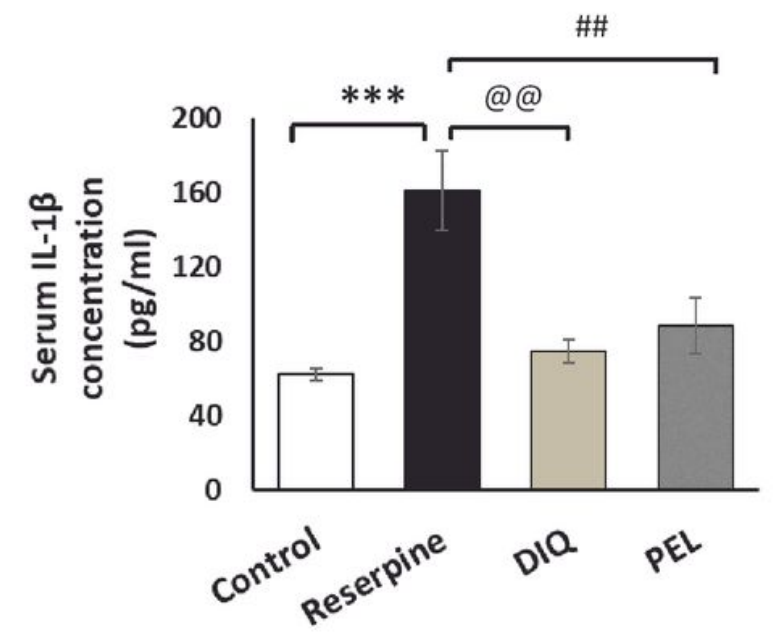

(b)

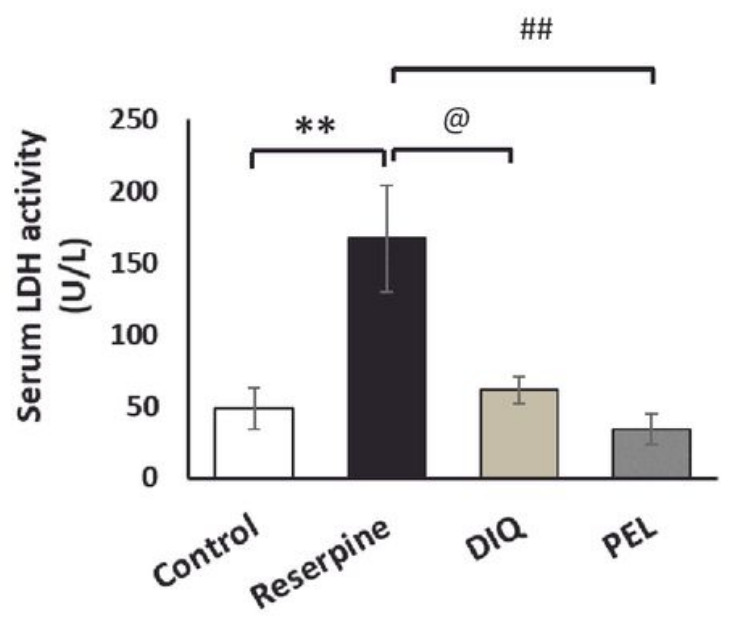

Figure 2

Effect of each of reserpine (5mg/ $\mathrm{kg})$, pelargonidin (PEL) (3mg/ $\mathrm{kg}$ ) and I,5-isoquinolinediol (DIQ) (10 $\mathrm{mg} / \mathrm{kg}$ ) on serum (a) Interleukin-1 $\beta$ (IL-1 $\beta$ ) concentration and (b) Lactate Dehydrogenase (LDH) activity in male rats. Results are represented as means of 6 observations per group \pm SEM. $\left({ }^{(*}\right)$ and ( $\left.{ }^{\star \star \star}\right)$ denote significant difference from the control group at $p<0.01$ and $p<0.001$; respectively. (\#) and (@@) denote significant difference from the reserpine-challenged group at $p<0.05$ and $p<0.01$; respectively 
(a)

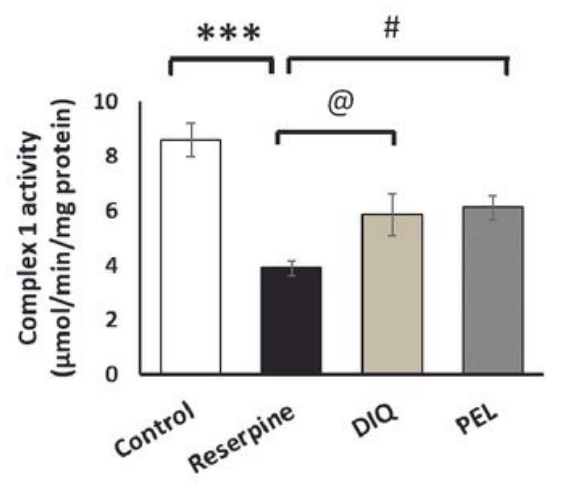

(c)

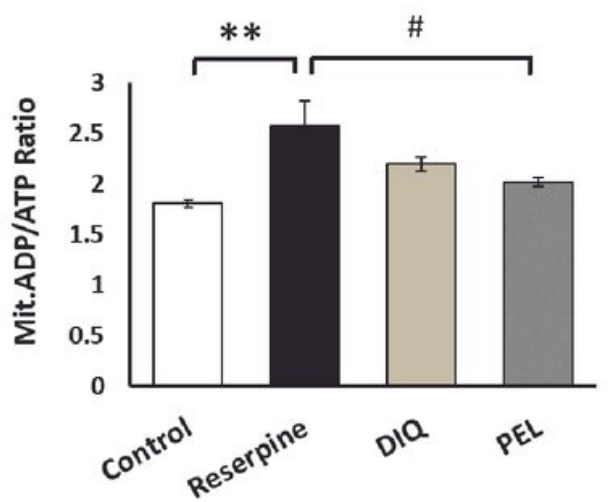

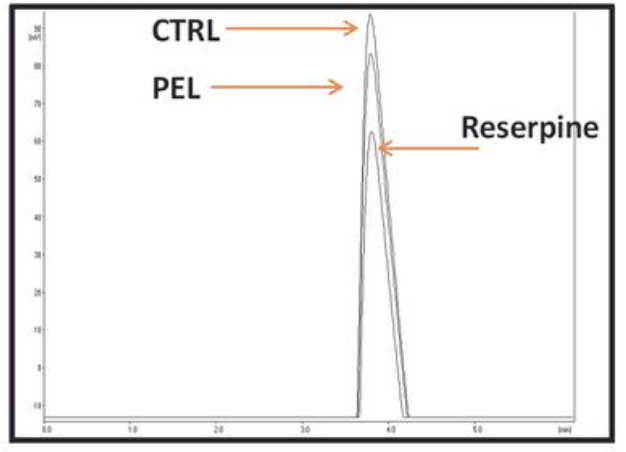

(b)

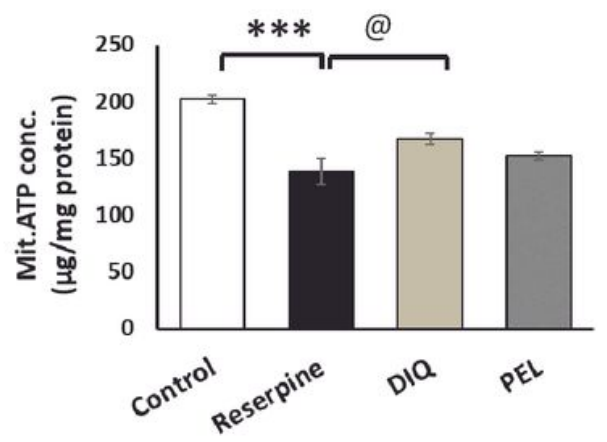

Figure 3

Effect of each of reserpine (5mg/ $\mathrm{kg})$, pelargonidin (PEL) $(3 \mathrm{mg} / \mathrm{kg})$ and I,5-isoquinolinediol (DIQ) (10 $\mathrm{mg} / \mathrm{kg}$ ) on brain mitochondrial (a) Complex-I activity, (b) Adenosine triphosphate (ATP) contents activity and (c) ADP/ATP ratio in male rats. Results are represented as means of 6 observations per group \pm SEM. $(* *)$ and $(* * *)$ denote significant difference from the control group at $p<0.01$ and $p<0.001$; respectively. (\#) and (@@) denote significant difference from the reserpine-challenged group at $p<0.05$ and $p<0.01$; respectively 

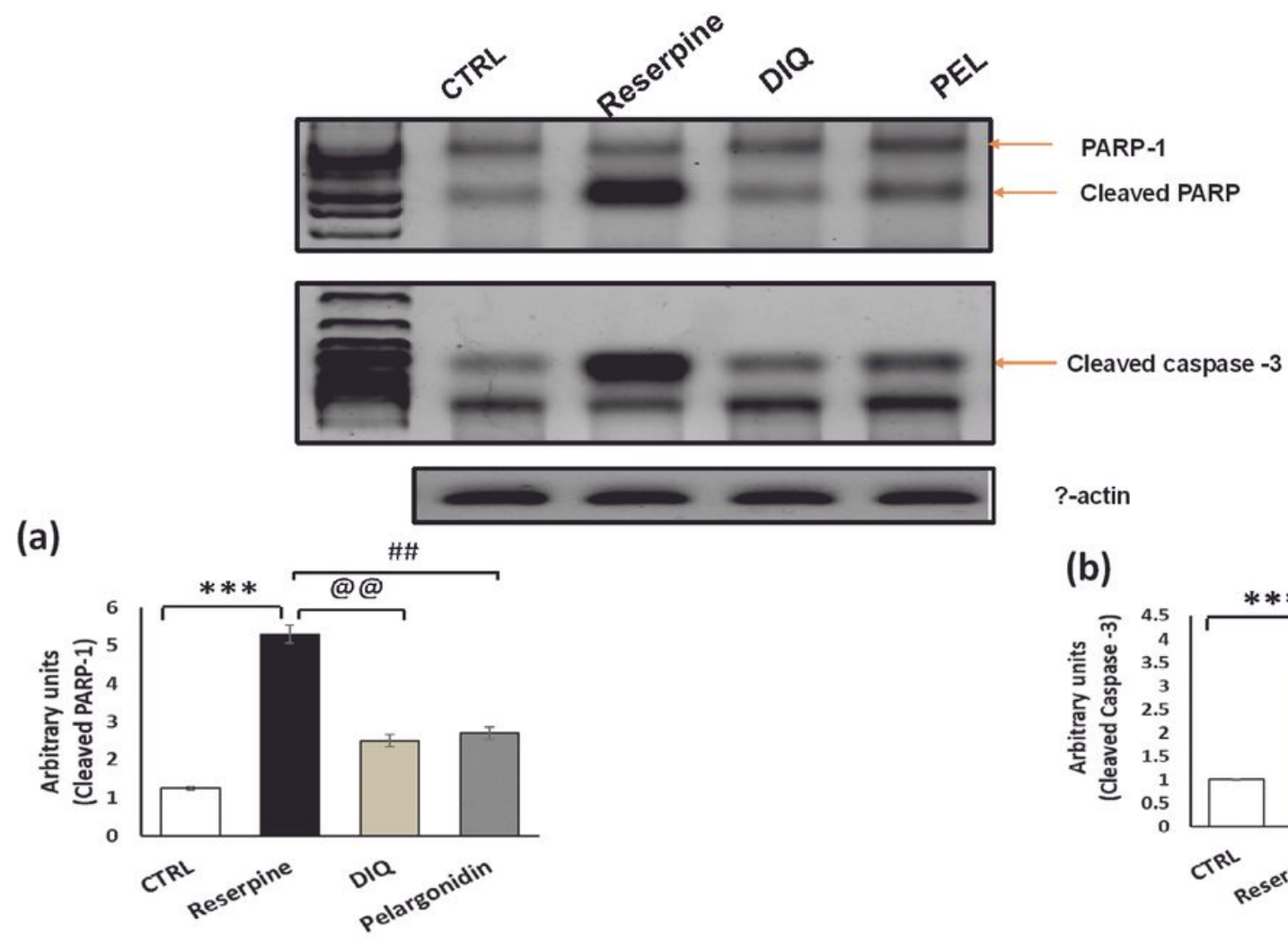

?-actin

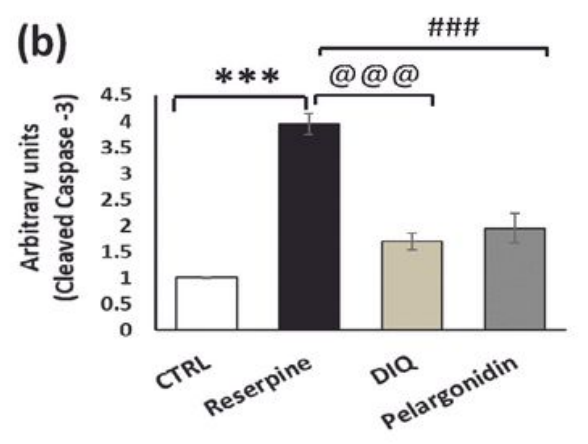

Figure 4

Effect of each of reserpine (5mg/ $\mathrm{kg}$ ), pelargonidin (PEL) $(3 \mathrm{mg} / \mathrm{kg})$ and I,5-isoquinolinediol (DIQ) (10 $\mathrm{mg} / \mathrm{kg}$ ) on (a) brain expression of cleaved PARP-1 and (b) Cleaved caspase-3. Blots of relative densitometry analysis of Cleaved PARP and Cleaved caspase-3 in male rats were normalized to $\beta$-actin densitometric values. Results are represented as means of 6 observations per group \pm SEM. ( $\left.{ }^{\star *}\right)$ and $(\star \star \star)$ denote significant difference from the control group at $p<0.01$ and $p<0.001$; respectively. (\#) and (@@) denote significant difference from the reserpine-challenged group at $p<0.05$ and $p<0.01$; respectively 


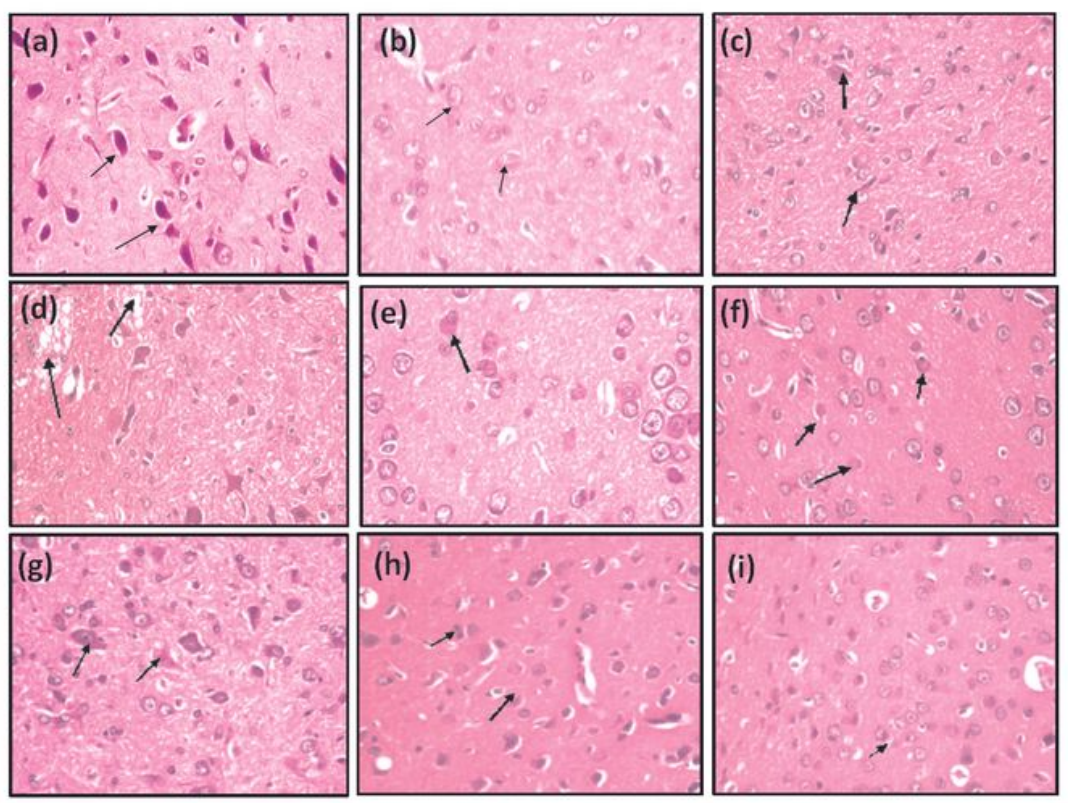

\section{Figure 5}

Microscopic images of rat brain tissues, A control group $(a, b)$ showing normal histological structure, Photomicrograph of substantia nigra showing (a) Perikaryon cells with short shrunken primary dendrites arrow (b) pyramidal or ovoid neuronal cells arrow. Brain tissues of reserpine-challenged rats(c,d,e and f) showed histological changes, Photomicrograph of substantia nigra showing (c) showing neurodegeneration and astrogliosis with microglial cell activation of nigral neurons arrow (H\&E X200) (d) showing neuronal loss, spongiosis arrow and gliosis (H\&E X200) (e) showing granular, pale-staining eosinophilic Lewy bodies (H\&EX400) (f) showing neuronal death, extracellular deposit Lewy bodies arrow (H\&E X400).Photomicrograph of substantia nigra of DIQ-treated rats showing (g) mild degenerative changes of neuronal cells and gliosis arrow (g) Swelling of neuronal cells with shorting of dendritic branches extra-cellular Lewy bodies arrow (H\&EX400) Photomicrograph of substantia nigra of PELtreated rats showing (h) Mild degenerative changes of neuronal cells with gliosis arrow (i) Swelling of neuronal cells with few number of Lewy bodies arrow (H\&EX400) 